\title{
Successes and challenges of the APP Coronagraph
}

\author{
Matthew A. Kenworthy ${ }^{1}$, Sascha Quanz ${ }^{2}$, Gilles Otten ${ }^{1}$, Tiffany \\ Meshkat $^{1}$, Johanan Codona ${ }^{3}$, Frans Snik ${ }^{1}$, Michael E. Meyer ${ }^{2}$, \\ Markus Kasper ${ }^{4}$ and Julien Girard ${ }^{5}$ \\ ${ }^{1}$ Leiden Observatory, Leiden University, P.O. Box 9513, 2300 RA Leiden, The Netherlands \\ email: kenworthy@strw.leidenuniv.nl \\ ${ }^{2}$ Institute for Astronomy, ETH Zurich, CH-8093 Zurich, Switzerland \\ ${ }^{3}$ Steward Observatory, 933 N. Cherry Avenue, Tucson, AZ 85721 \\ ${ }^{4}$ European Southern Observatory, Garching bei Munchen, Germany \\ ${ }^{5}$ European Southern Observatory, Vitacura, Cassilla 19001, Santiago, Chile
}

\begin{abstract}
.
The Apodizing Phase Plate (APP) coronagraph has been used to image the exoplanet $\beta$ Pictoris b and the protoplanet candidate around HD 100546, and is currently in use in surveys with $\mathrm{NaCo}$ at the VLT. Its success is due to its tolerance to tip-tilt pointing errors in current $\mathrm{AO}$ systems, which degrade the performance of nearly all other coronagraphs. Currently the sensitivity of the APP is limited by non-common path errors in the science camera systems and by its chromatic behaviour. We present the achromatized Vector APP coronagraph and address how we will measure and minimise non-common path errors with Focal Plane Wavefront Sensing algorithms.
\end{abstract}

Keywords. instrumentation: high angular resolution, stars: planetary systems, stars: individual ( $\beta$ Pic, HD 100546)

\section{Introduction}

Coronagraphs are used to suppress the diffraction halo around bright stars, revealing the presence of faint companions (brown dwarfs and planets) next to their primary star. There are many coronagraph designs that work at small inner working angles (Guyon et al. 2006) and continuing technological developments are ongoing (for a recent review see Mawet et al. 2012).

The Apodizing Phase Plate (APP) Coronagraph is a pupil-plane optic that is a realization of Phase Apodization Coronagraphy (Codona \& Angel 2004; Codona et al. 2006). In common with other pupil plane reformatters (Carlotti et al. 2012; Carlotti 2013), it is therefore relatively insensitive to residual tip-tilt motions in the focal plane of the science camera from residual atmospheric correction and from vibrations in the telescope superstructure. A first light demonstrator on the MMTO $6.5 \mathrm{~m}$ telescope at $\lambda=4.85 \mu \mathrm{m}$ (Kenworthy et al. 2007), was followed by an $L^{\prime}$ band APP on the Very Large Telescope (Kenworthy et al. 2010a,c; Girard et al. 2010) and most recently at $L^{\prime}$ band on the Large Binocular Telescope in LMIRCam (Wilson et al. 2008; Kenworthy et al. 2010b; Leisenring et al. 2012). Characterizations of planets include $\beta$ Pictoris b (Quanz et al. 2010) and the discovery of a low mass companion to HD 100546 (Quanz et al. 2013), along with its inclusion in several VLT surveys for extrasolar planets around nearby stars with debris disks (Meshkat et al. 2013) and A/F stars (Quanz et al. 2011). 


\section{Current Performance and Future Directions}

APP observations have placed strong constraints on low mass objects at Solar System scales around Fomalhaut (Kenworthy et al. 2013), typically with contrasts of 12 mag at 0.4 arcseconds. The refinement of Principal Component Analysis (PCA) techniques (Amara \& Quanz 2012; Soummer et al. 2012) lead to an additional $~ 0.5$ mag of sensitivity (see Meshkat et al. these proceedings; Meshkat et al. (2013) submitted).

The performance of the APP coronagraph is limited by (i) the non-common path aberrations (NCPA) between the AO camera and the wavefront sensor path in the science instrument, and (ii) its chromatic design. With the APP we will use Phase Sorting Interferometry (Codona \& Kenworthy 2013) which uses phase diversity from the AOcorrected atmosphere to sense NCPA. Another development is the Vector APP (VAPP), an implementation of the APP that uses multiple stacked layers of polarization phase plates to provide a broadband wavelength solution (Snik et al. 2012) that also provides two simultaneous and complementary PSFs with the dark D region on opposing sides around the star. New versions of this optic are currently being characterized at Leiden Observatory, and the APP is being considered as the baseline coronagraph for ERIS, the new thermal infrared imager for the VLT, and for METIS, the thermal infrared imager and spectrograph for the European ELT.

\section{References}

Amara A. \& Quanz S. P., 2012, MNRAS, 427, 948

Carlotti A., 2013, A\&A, 551, A10

Carlotti A., Kasdin N. J., Vanderbei R. J., \& Delorme J.-R., 2012, SPIE 8442.

Codona J. L. \& Angel R., 2004, ApJ, 604, L117

Codona J. L. \& Kenworthy M., 2013, ApJ, 767, 100

Codona J. L., Kenworthy M. A., Hinz P. M., Angel J. R. P., \& Woolf N. J., 2006, SPIE 6269, pp. $62691 \mathrm{~N}$

Girard J. H. V., et al., 2010, SPIE 7736

Guyon O., Pluzhnik E. A., Kuchner M. J., Collins B., \& Ridgway S. T., 2006, ApJS, 167, 81

Kenworthy M., Quanz S., Meyer M., Kasper M., Girard J., Lenzen R., Codona J., \& Hinz P., 2010a, The Messenger, 141, 2

Kenworthy M. A., Codona J. L., Hinz P. M., Angel J. R. P., Heinze A., \& Sivanandam S., 2007, ApJ, 660, 762

Kenworthy M. A., Hinz P. M., Codona J. L., Wilson J. C., Skrutskie M. F., \& Solheid E., 2010b, SPIE 7734

Kenworthy M. A., Meshkat T., Quanz S. P., Girard J. H., Meyer M. R., \& Kasper M., 2013, ApJ, 764, 7

Kenworthy M. A., Quanz S. P., Meyer M. R., Kasper M. E., Lenzen R., Codona J. L., Girard J. H., \& Hinz P. M., 2010c, SPIE 7735

Leisenring J. M., et al., 2012, SPIE 8446

Mawet D., et al., 2012, SPIE 8442

Meshkat T., Kenworthy M. A., Su K. Y., \& Bailey V. P., 2013, AAS 221, 149.28

Quanz S. P., Amara A., Meyer M. R., Kenworthy M. A., Kasper M., \& Girard J. H., 2013, ApJ, $766, \mathrm{~L} 1$

Quanz S. P., Kenworthy M. A., Meyer M. R., Girard J. H. V., \& Kasper M., 2011, ApJ, 736, L32

Quanz S. P., Meyer M. R., Kenworthy M. A., Girard J. H. V., Kasper M., Lagrange A.-M., Apai D., Boccaletti A., Bonnefoy M., Chauvin G., Hinz P. M., \& Lenzen R., 2010, ApJ, 722, L 49

Snik F., Otten G., Kenworthy M., Miskiewicz M., Escuti M., Packham C., Codona J., 2012, SPIE 8450

Soummer R., Pueyo L., \& Larkin J., 2012, ApJ, 755, L28

Wilson J. C., Hinz P. M., Skrutskie M. F., Jones T., Solheid E., Leisenring J., Garnavich P., Kenworthy M., Nelson M. J., \& Woodward C. E. 2008, SPIE 7013 